\title{
Synthesis and Characterization of $\mathrm{SiO}_{2} @ \mathrm{CNTs}$ Microparticles: Evaluation of Microwave-Induced Heat Production
}

\author{
Panagiotis Kainourgios, Ioannis A. Kartsonakis * $\mathbb{D}$ and Costas A. Charitidis *(D) \\ Research Unit of Advanced, Composite, Nano-Materials and Nanotechnology, School of Chemical Engineering, \\ National Technical University of Athens, 9 Heroon Polytechniou Street, Zographos, GR-15773 Athens, Greece; \\ pkainourgios@chemeng.ntua.gr \\ * Correspondence: ikartso@chemeng.ntua.gr (I.A.K.); charitidis@chemeng.ntua.gr (C.A.C.); \\ Tel.: +30-210-772-4046 (C.A.C.)
}

Citation: Kainourgios, P.;

Kartsonakis, I.A.; Charitidis, C.A.

Synthesis and Characterization of $\mathrm{SiO}_{2} @ \mathrm{CNTs}$ Microparticles:

Evaluation of Microwave-Induced Heat Production. Fibers 2021, 9, 81. https://doi.org/10.3390/ fib9120081

Academic Editor: Ramiro Rafael Ruiz Rosas

Received: 4 November 2021

Accepted: 1 December 2021

Published: 3 December 2021

Publisher's Note: MDPI stays neutral with regard to jurisdictional claims in published maps and institutional affiliations.

\begin{abstract}
This study was focused on the growth of multi-walled carbon nanotubes (MWCNTs) on iron chloride-functionalized silica microspheres. In addition, the microwave absorption potential and the subsequent heat production of the resulting structures were monitored by means of infrared thermometry and compared with pristine commercially available MWCNTs. The functionalized silica microparticle substrates produced MWCNTs without any amorphous carbon but with increased structural defects, whereas their heat production performance as microwave absorbents was comparable to that of the pristine MWCNTs. Two-minute microwave irradiation of the $\mathrm{SiO}_{2} @ \mathrm{CNTs}$ structures resulted in an increase in the material's temperature from ambient temperature up to $173{ }^{\circ} \mathrm{C}$. This research puts forward a new idea of charge modulation of MWCNTs and sheds light on an investigation for the development of bifunctional materials with improved properties with respect to efficient microwave absorbance.
\end{abstract}

Keywords: microwave irradiation; carbon nanotubes; chemical vapor deposition; nanostructured powders; Raman

\section{Introduction}

Currently, reduction, recycling and reuse are crucial parameters in order to tackle waste problems and to move from linear economic processes and systems towards a more circular economy [1]. Therefore, material development methods progressively lean towards green synthesis procedures, aiming at recycling strategies as well as the reusability of raw materials. Furthermore, in the industries related to composite and coating manufacture, there is a growing demand for reclaimable materials, which opposes a serious challenge due to the permanent nature of the bonding between the matrix and reinforcing material [1]. For many years, the term "debonding" has been addressed as a form of material failure, where loss of adhesion occurs between the matrix and filler [2]. On the other hand, recent studies propose alternatives for the fabrication of coatings where debonding-on-demand is being described as a mechanism to separate the two phases of a composite material or a coated surface via the application of an external stimulus. From the chemical perspective, such strategies include the synthesis of crosslinked polymers utilizing photo-initiators for triggering mechanisms [3-5]. In addition, the use of nanoparticles (NPs) could potentially address this challenge due to their unique intrinsic properties [6-8].

Notwithstanding, CNTs have attracted significant attention as filler materials because of their unique physical properties [9]. One of the most important properties of CNTs is their response to microwave irradiation by producing heat. Imholt et al. previously described the emission of light as well as the production of intense heat when singlewalled carbon nanotubes (SWCNTs) were exposed to microwave irradiation [10]. For these reasons, several vibration analyses have been performed related to the number of walls of the nanotubes. In the work of Strozzi et al., linear vibrations of triple-walled 
carbon nanotubes were investigated in order to determine the effect of the geometry and boundary conditions on the natural frequencies of the nanotubes [11]. In the study of He et al., it was found that the van der Waals interaction influences the radial vibration of small-radius multi-walled carbon nanotubes (MWCNTs) [12]. Moreover, the mechanical behavior of MWNTs as single entities, as well as their effectiveness as load-bearing entities in nanocomposite materials, was investigated by Pantano et al., focusing on the use of bent MWNTs of different diameters and numbers of walls [13]. Although the exact mechanism of microwave absorbance is still under vigorous research, its interaction is also being studied in terms of microwave-assisted synthesis, functionalization or purification, whereas in all cases examined, the production of heat is a common aspect [14].

Purification of MWCNTs includes several steps and can be performed via a variety of procedures that emphasize the removal of the catalytic particles which are important for the growth of CNTs. These particles are transition metal nanoparticles, in most cases iron oxide nanoparticles. In the work of Wadhawan et al., the microwave absorption of purified and unpurified SWCNTs was examined. The outcome of their study revealed an increase in heating in the unpurified sample compared to the purified one. They also found that the unpurified SWCNTs also emitted light, a result that clearly denoted the evidence of heat production after microwave irradiation, as well as the hypothesis that iron oxide nanoparticles contribute to microwave absorption [15]. The exceptional properties of CNTs are attributed to their defect-free graphene layers, including microwave absorption; the defect-free surface, however, renders the material chemically inert and therefore opposes a challenge towards practical applications such as homogenous dispersions of CNTs in thermoplastic matrices [16].

On the other hand, silica nanoparticles produced by the Stöber process, can be surface functionalized in order to be homogenously dispersed in a variety of media [17]. In the work of Wang et al., a core-shell three-dimensional hierarchical structure based on $\mathrm{Fe}_{3} \mathrm{O}_{4}$ was fabricated in a multi-component glass system that was able to exhibit effective microwave absorption [18]. In addition, Green et al. produced doped silica nanoparticles, which, according to their findings, exhibited excellent microwave absorption performance [19]. From the aforementioned research outcomes, it is evident that both silica and iron oxide have potential microwave absorption properties.

In this study, silica nanoparticles were fabricated and surface functionalized with iron chloride to provide substrates/catalytic particles for the growth of carbon nanotubes. The CNTs' growth was studied morphologically (and qualitatively) by scanning electron microscopy (SEM), quantitatively by means of thermogravimetric analysis (TGA) and qualitatively utilizing Raman spectroscopy to identify the $\mathrm{I}_{\mathrm{d}} / \mathrm{I}_{\mathrm{g}}$ ratio. The as-produced nanomaterials were subsequently microwave irradiated, and their heat production was estimated by incorporating infrared thermometry. The added value of this work lies in the fact that the individual properties of each component, namely, surface functionalization and microwave absorption for silica nanoparticles and MWCNTs, respectively, render this hybrid material a potential debonding agent in the composite and coating industries. The resulting hybrid structures present both microsilica properties (high surface area, surface functionalization/chemical affinity with polymer matrices) and heat production via microwave absorption due to the MWCNT counterpart and therefore can be considered a novel material in the composite and coating industries.

\section{Materials and Methods}

\subsection{Materials}

Absolute ethanol 99.5\% and ammonium hydroxide were purchased from Acros Organics, whereas tetraethyl orthosilicate (TEOS) and iron chloride (II) tetrahydrate $99 \%$ were purchased from Sigma Aldrich (Saint Louis, MI, USA) and used without any further purification. For the chemical vapor deposition (CVD) experiment, acetylene was used as the carbon source, whereas argon and hydrogen were used as inert and reducing agents, respectively. Finally, apart from the laboratory synthesis of MWCNTs, pris- 
tine commercial MWCNTs were also purchased from Hongwu International Group Ltd. (Guangzhou, China).

\subsection{Synthesis of $\mathrm{SiO}_{2} @ \mathrm{CNTs}$}

The synthesis of MWCNTs on the surface of colloidal silica, $\mathrm{SiO}_{2} @ \mathrm{CNTs}$, was accomplished by a three-step process: (i) formation of colloidal silica by means of sol-gel (Stöber method) [17], (ii) functionalization of the hydroxyl groups of colloidal silica with iron chloride and iii) heat treatment of the functionalized $\mathrm{SiO}_{2}$ at $700{ }^{\circ} \mathrm{C}$ under an oxygen atmosphere to calcinate the sample and form iron oxide nanoparticles, with subsequent reduction under $\mathrm{H}_{2}$ flow in the presence of acetylene as a carbon source to initiate the growth of carbon nanotubes.

Briefly, $100 \mathrm{~mL}$ of ethanol, $11 \mathrm{~mL}$ of $\mathrm{H}_{2} \mathrm{O}, 6 \mathrm{~mL}$ of TEOS and $8 \mathrm{~mL}$ of ammonia as a catalyst were placed in a $250 \mathrm{~mL}$ round-bottom flask. The mixture was left under vigorous stirring for $5 \mathrm{~h}$ to produce silica microspheres with average diameters of $350 \mathrm{~nm}$. Subsequently, centrifugation and rinsing with deionized water followed. The as-received white powder was left to dry in a desiccator.

Functionalization of the hydroxyl groups of colloidal silica was conducted with iron chloride aqueous solution by means of electrostatic interaction. The mechanism of the electrostatic interaction is realized by the interaction of the negatively charged hydroxyl groups of silica with the positively charged $\mathrm{Fe}^{+}$ions. This technique was demonstrated in our previous work utilizing negatively charged polymethacrylic acid nanoparticles and silver ions [20].

The silica nanospheres were dispersed in an aqueous solution of $10 \mathrm{mM}$ iron chloride tetrahydrate $99 \%$ for $2 \mathrm{~h}$ in order for $\mathrm{Fe}^{+}$ions to electrostatically attach to the hydroxyl groups of silica. Centrifugation and rinsing followed, and the as-received orange powder was dried in a desiccator.

After the functionalized nanospheres were dried, they were grinded into a fine powder and placed on top of a silicon wafer. Then, the wafer was placed inside a horizontal CVD furnace that was set to $700{ }^{\circ} \mathrm{C}$ under an oxygen atmosphere to calcinate the sample and form iron oxide nanoparticles. Subsequently, a reduction process followed under $\mathrm{H}_{2}$ flow $(220 \mathrm{sccm})$ for $10 \mathrm{~min}$, and then under acetylene flow $(300 \mathrm{sccm})$ for $10 \mathrm{~min}$ as a carbon source inserted into the system to initiate the growth of MWCNTs on the colloidal silica surface. Further purification (removal of the iron oxide nanoparticles by chemical oxidation) of the as-produced MWCNTs was not realized in order to avoid introducing further defects in the MWCNTs.

\subsection{Characterization}

The materials' surface morphology was estimated via scanning electron microscopy (SEM) using a PHILIPS Quanta Inspect (FEI Company, Hillsboro, OR, USA) microscope with a W (tungsten) filament $25 \mathrm{KV}$ equipped with a EDAX GENESIS (Ametex Process \& Analytical Instruments, Pittsburgh, PA, USA). Micro-Raman measurements were performed using a Renishaw inVia spectrometer working in a backscattering configuration and equipped with a near-infrared diode laser emitting at $530 \mathrm{~nm}$. The spectra were recorded by focusing the laser beam on the sample surface and adjusting the light power so that $1 \mathrm{~mW}$ was provided for a spot of about $1 \mu \mathrm{m}$ diameter. Thermogravimetric analysis (TGA) was performed on a TGA Netch STA 449 Jupiter. Samples were heated from ambient temperature to $900{ }^{\circ} \mathrm{C}$ with a heating rate of $10^{\circ} \mathrm{C} / \mathrm{min}$ under an $\mathrm{O}_{2}$ atmosphere. A Malvern Zetasizer nano zs apparatus was utilized for measuring the size, polydispersity and surface charge of the silica microsphere by dynamic light scattering (DLS). In addition, a Cary 630 FT-IR Spectrometer was utilized to perform the FT-IR measurements. Each sample was grinded into a powder and mixed with $\mathrm{KBr}$ to form pellets for the measurements in the range of 400 to $4500 \mathrm{~cm}^{-1}$.

Microwave absorption of materials is a complex scientific field that requires a wellestablished profile of the electromagnetic properties of the tested material in order to be 
assessed with precision. This work was focused on the synthesis and characterization of a novel material with potential microwave absorption properties; the method applied for the microwave absorption is straightforward, and the presented results serve as an indication and not as absolute values. The microwave absorption was measured utilizing a microwave instrument (magnetron $700 \mathrm{~W}$ operating at $2.45 \mathrm{GHz}$ ), whereas the heat produced was assessed utilizing an FLIR C3-X thermal camera. For the microwave absorption assessment, 4 samples were tested: an empty vial as a control, plain silica microspheres, pristine commercial MWCNTs, with a mass corresponding to a $20 \%$ mass percentage of the hybrid structures, and the final hybrid structure, which, from this point on, will be referred to as $\mathrm{SiO}_{2} @ \mathrm{CNTs}$.

\section{Results and Discussion}

\subsection{Morphological Characterization}

The morphological evaluation of $\mathrm{SiO}_{2} @ \mathrm{CNTs}$ was accomplished via SEM, whereas DLS was used to correlate the size results with SEM analysis; the results are exhibited in Figures 1 and 2, respectively. Figure 1a depicts the pristine silica microspheres with an average diameter at $350 \mathrm{~nm}$; these results are in agreement with the size measurements obtained from DLS, Figure 2a, which reveal an average hydrodynamic radius of $400 \mathrm{~nm}$. The $50 \mathrm{~nm}$ difference can be attributed to the strong negative charge of silica microspheres $(-40 \mathrm{mV})$, as seen in the zeta potential measurement in Figure 2b. Furthermore, the formation of iron oxide nanoparticles on the surface of silica microspheres prior to the growth of the MWCNTs is demonstrated in Figure 1b,c. It can be seen in Figure 1c that iron oxide nanoparticles with a diameter range from 15 to $21 \mathrm{~nm}$ were grown on the silica microspheres, after functionalizing the latter with iron chloride and subsequent thermal oxidation at $700{ }^{\circ} \mathrm{C}$. However, the aforementioned observation is not evident for all the silica microspheres since the electrostatic attachment is of a dynamic nature, affected by the surface charge of the silica particles, the concentration of iron chloride and the experimental conditions such as the temperature and stirring time. Energy-dispersive $\mathrm{X}$-ray analysis (EDS) was performed on the silica microspheres prior to the growth of MWCNTs; the results are illustrated in Figure 3. It can be observed that the elemental analysis affirms the presence of elemental iron at 6.3 and $0.7 \mathrm{keV}$. However, it should be mentioned that the presence of carbon and gold is attributed to the SEM sample preparation: carbon due to the use of carbon tape for sample adhesion, and gold due to the gilding process in order for the sample to become more conductive. Therefore, carbon and gold were excluded from the elemental quantification and the corresponding calculation of the atomic percentages that are illustrated in Figure 3.

Figure $1 \mathrm{~d}-\mathrm{f}$ exhibit the surface iron oxide-functionalized silica microspheres after the growth of MWCNTs. Starting from smaller magnifications, Figure 1d, moving to larger ones, Figure 1f, the growth of MWCNTs with a random orientation and an average thickness of $50 \mathrm{~nm}$ can be observed. As in the case of iron oxide nanoparticles, some silica microspheres do not present any MWCNT growth, Figure 1e,f. This outcome can be potentially attributed to partial functionalization of the initial silica microspheres due to the dynamic nature of the electrostatic bonding of Fe ions to the negatively charged microspheres [21,22]. Nevertheless, both the iron oxide nanoparticles and the as-produced MWCNTs exhibit an extremely narrow nanoparticle size and MWCNT thickness distribution, thus further supporting the effect of the catalytic nanoparticle size in the growth of MWCNTs [21,23]. 


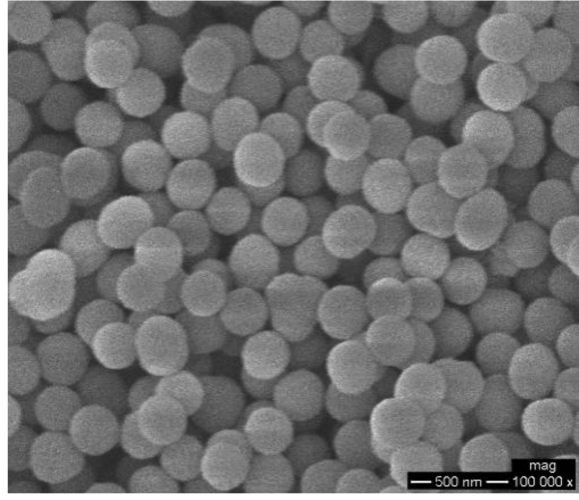

(a)

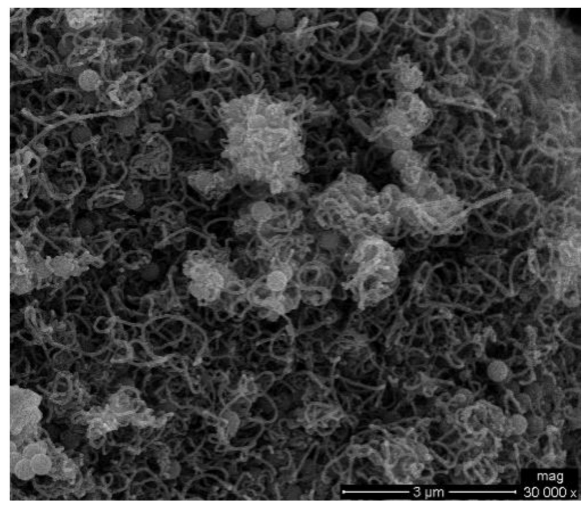

(d)

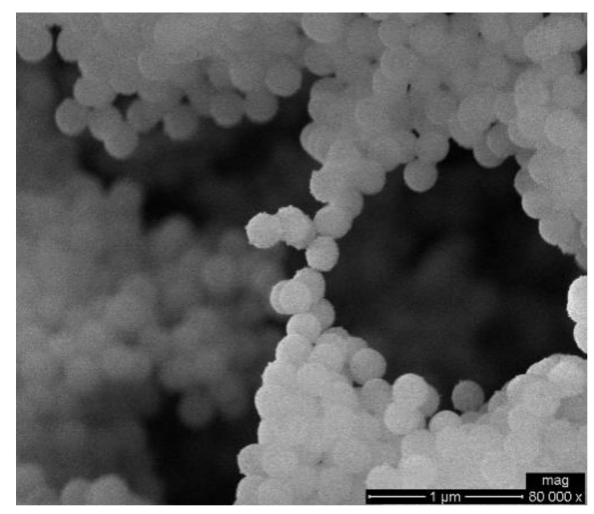

(b)

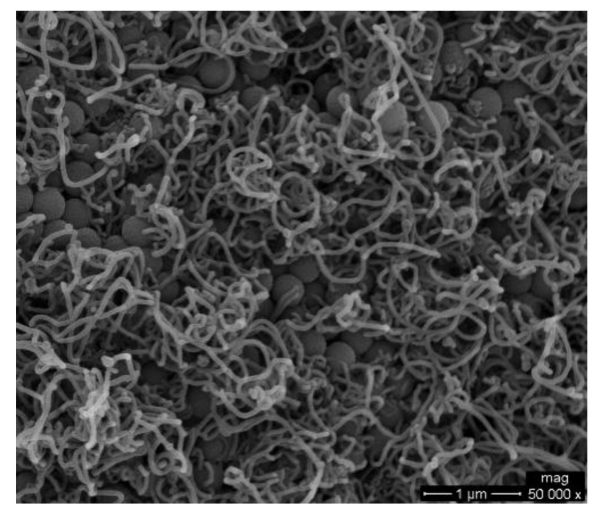

(e)

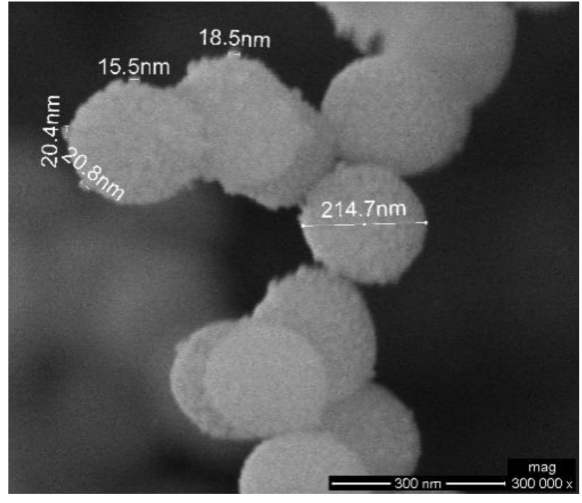

(c)

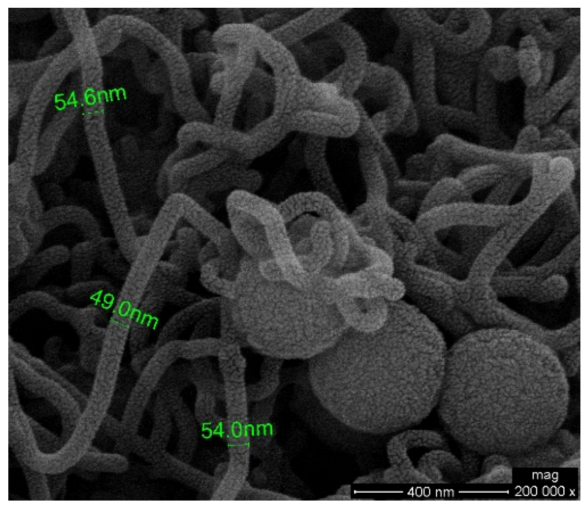

(f)

Figure 1. SEM images of (a) silica microspheres; $(\mathbf{b}, \mathbf{c})$ silica microspheres with iron oxide nanoparticles at different magnifications; and (d-f) $\mathrm{SiO}_{2} @ \mathrm{CNTs}$ at different magnifications.

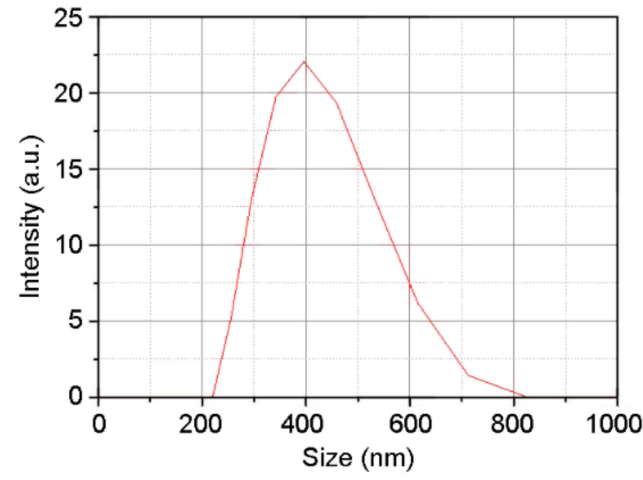

(a)

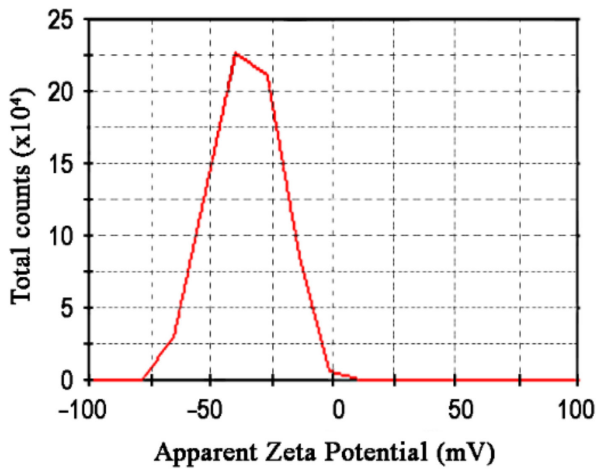

(b)

Figure 2. Dynamic light scattering of silica samples: (a) hydrodynamic radius; (b) zeta potential. 


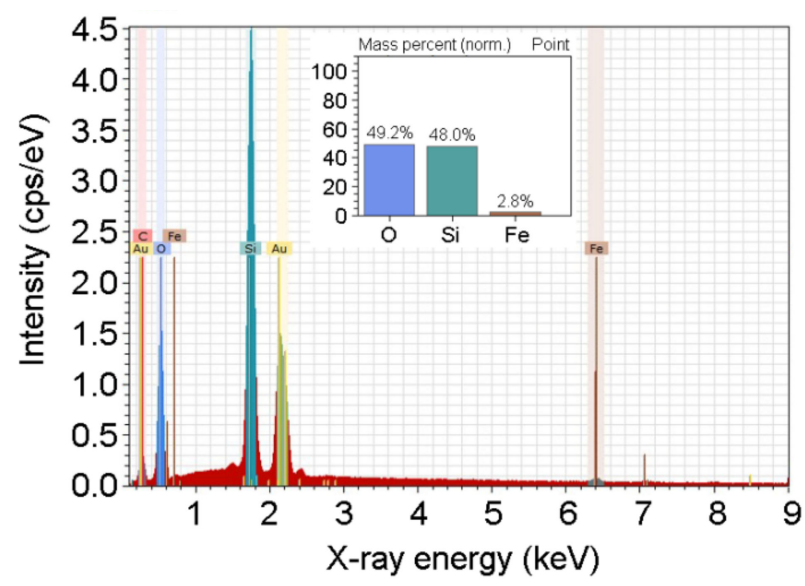

Figure 3. EDS analysis of the $\mathrm{Fe}^{+}$-functionalized silica microspheres.

\subsection{Structural and Thermal Analyses}

Raman and TGA analyses were performed for the quantitative determination and qualitative identification, respectively, of the structural defects of MWCNTs on the $\mathrm{SiO}_{2} @ \mathrm{CNTs}$ hybrid structures. Figure 4 presents the results of the TGA analysis. It is shown that at approximately $100{ }^{\circ} \mathrm{C}$, a minor mass increase can be observed ( $\left.0.8 \mathrm{wt} . \%\right)$, which is attributed to the initial oxidation. According to Mansfield et al., amorphous carbon thermal degradation occurs between 200 and $400{ }^{\circ} \mathrm{C}$. Therefore, taking into account that no mass loss can be seen in this temperature range, it can be assumed that the $\mathrm{SiO}_{2} @ \mathrm{CNTs}$ sample has no amorphous carbon $[24,25]$. The thermal degradation of the sample initiates at $463^{\circ} \mathrm{C}$ and ends at $670{ }^{\circ} \mathrm{C}$, having lost $20 \mathrm{wt} . \%$ of its total mass. From there on, the mass remains constant up to $900^{\circ} \mathrm{C}$, where the measurement is finished. This mass loss is attributed to the thermal oxidation of MWCNTs, which occurs above $450{ }^{\circ} \mathrm{C}$ in air [26]. According to the literature, above the aforementioned temperature under an oxygen atmosphere, the graphite layers of MWCNTs become unstable and begin to degrade; this outcome has been observed during the heating of MWCNTs at various heating rates [27]. Evidently, the TGA measurements revealed a $20 \mathrm{wt} . \%$ MWCNTs content with minor to no amorphous carbon content.

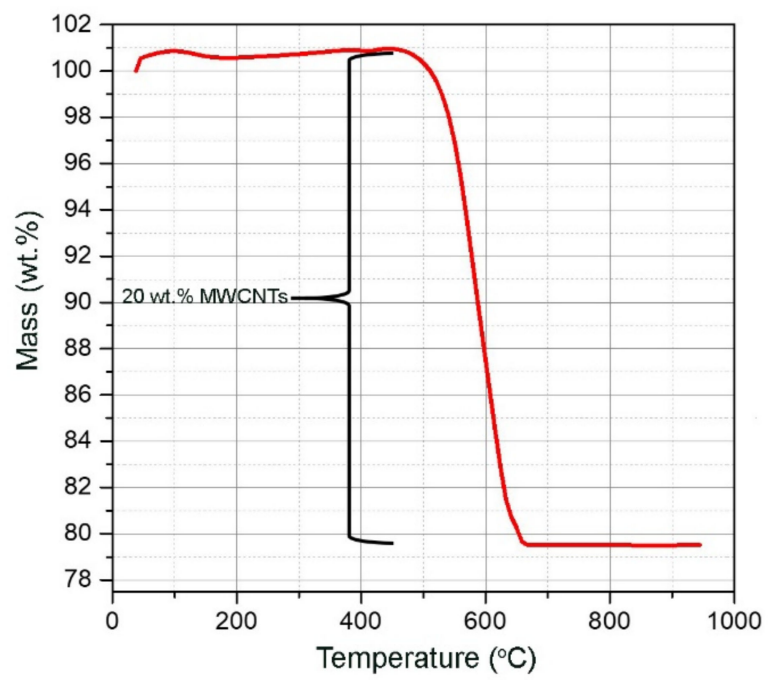

Figure 4. TGA curve of the thermal decomposition of $\mathrm{SiO}_{2} @ \mathrm{CNT}$ s.

Although the $\mathrm{SiO}_{2} @ \mathrm{CNTs}$ were free of amorphous carbon, Raman analysis (Figure 5a) revealed significant structural defects in the carbon structures judging by the calculation of the $\mathrm{I}_{\mathrm{d}} / \mathrm{I}_{\mathrm{g}}$ ratio $\left(\mathrm{I}_{\mathrm{d}} / \mathrm{I}_{\mathrm{g}}=2.0\right)$. In addition to the $\mathrm{D}$ and $\mathrm{G}$ bands, the $2 \mathrm{D}$ (or $\mathrm{D}^{\prime}$ ) band is also present, along with the $\mathrm{D}+\mathrm{G}$ band, thus further justifying the presence of MWCNTs in 
the sample. On the other hand, the Raman spectrum of the pristine commercial MWCNTs, Figure $5 \mathrm{~b}$, presents the same bands; however, the D band at approximately $1350 \mathrm{~cm}^{-1}$, which is correlated with defects in the CNTs' graphitic structures, is significantly lower in area compared to the $\mathrm{SiO}_{2} @ C N T s$ spectrum, Figure $5 \mathrm{a}$, as well as the $\mathrm{I}_{\mathrm{d}} / \mathrm{I}_{\mathrm{g}}$ ratio $(0.6)$, whereas the 2D band is notably stronger. It is evident, from the Raman analysis, that the commercial MWCNTs sample exhibits a more refined structure in terms of defects in the graphitic layers.

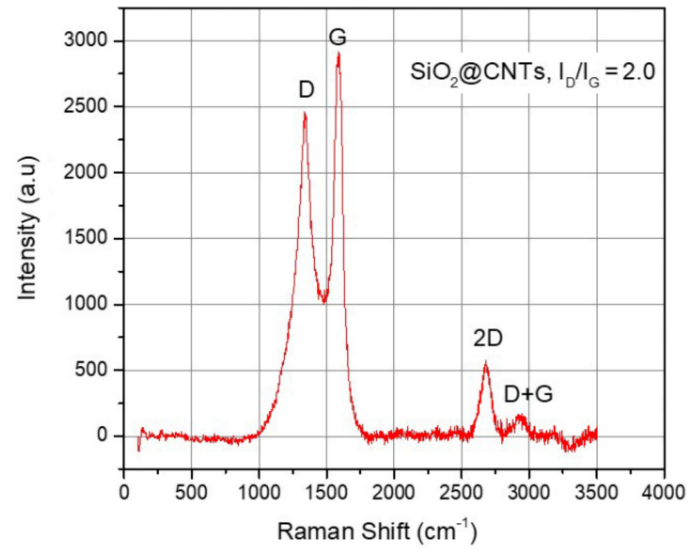

(a)

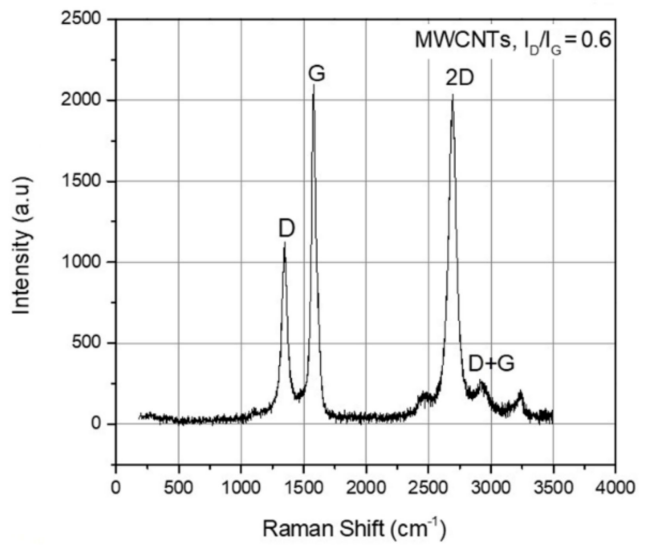

(b)

Figure 5. Raman shift of (a) $\mathrm{SiO}_{2} @ C N T s$ and (b) pristine commercial MWCNTs.

To further support the presence of MWCNTs on the as-produced silica surface, XRD measurements took place in order to identify any crystal structures present on the sample as evidence of MWCNTs; the results are presented in Figure 6. The XRD spectrum exhibits one broad and one sharp peak located at approximately 25 degrees and 43 degrees, respectively. According to the literature [28], amorphous nano-silica exhibits a broad peak with a center at approximately 25 degrees, which agrees with our findings since in the TGA measurements, it was shown that the sample consists primarily of silica (80\%). Nevertheless, MWCNTs exhibit two main peaks, the first one located at 23 degrees and the second one at 43 degrees, responsible for the diffraction of the crystal graphitic planes (002) and (100), respectively $[29,30]$. Although the first peak is most likely overlapped by the strong diffraction of the amorphous silica, the second peak at 43 degrees is a sharp peak with a strong intensity, clearly denoting evidence of crystal structures in the $\mathrm{SiO}_{2} @ \mathrm{CNTs}$ sample.

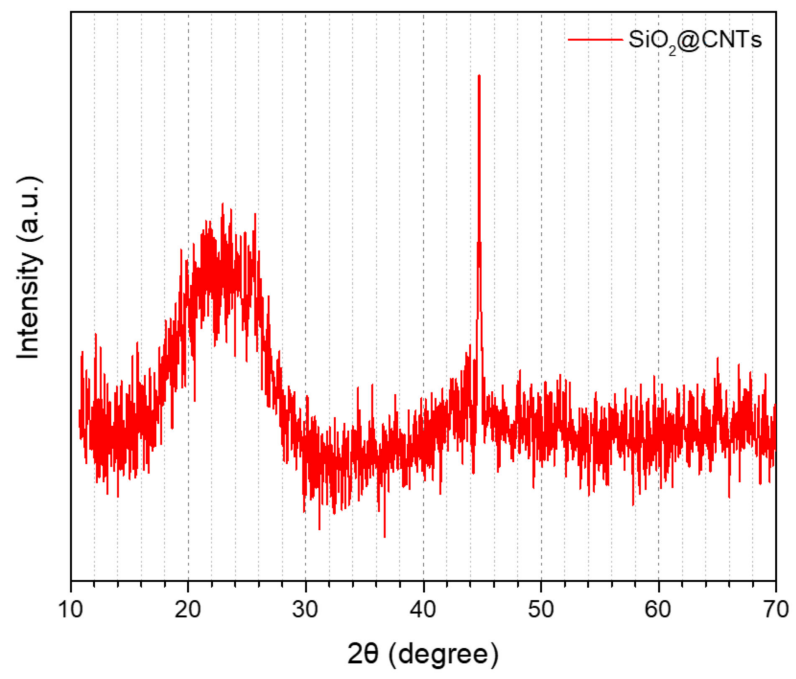

Figure 6. XRD spectrum of $\mathrm{SiO}_{2} @ \mathrm{CNTs}$. 
According to the literature, the catalytic activity for the growth of MWCNTs is provided by transition metal particles-in our case, elemental iron [31,32]. For this reason, after the functionalization of the hydroxyl groups of colloidal silica with iron chloride aqueous solution by means of electrostatic interaction, the produced iron oxide nanoparticles undergo reduction to elemental iron during the reduction process with $\mathrm{H}_{2}$ at $700{ }^{\circ} \mathrm{C}$. FT-IR analysis was performed on the pristine silica microspheres and on the silica microspheres after the functionalization with iron chloride and their calcination at $700{ }^{\circ} \mathrm{C}$ $\left(\mathrm{Fe}^{+}\right.$-functionalized silica microspheres). The results are illustrated in Figure 7. Figure 7a exhibits the FT-IR spectrum of the pristine silica microspheres; the absorption band at $810 \mathrm{~cm}^{-1}$ is attributed to $\mathrm{Si}-\mathrm{O}-\mathrm{Si}$ stretching. The absorption bands at $1070 \mathrm{~cm}^{-1}$ and $950 \mathrm{~cm}^{-1}$ are assigned to the siloxane vibration, whereas the bands at $3200-3500 \mathrm{~cm}^{-1}$ and at $1625 \mathrm{~cm}^{-1}$ are attributed to the $\mathrm{O}-\mathrm{H}$ stretching band of either the hydroxyl groups of the silica microspheres or the water molecules present in the sample [33]. Figure $7 \mathrm{~b}$ depicts the FT-IR absorption spectrum of the silica microspheres after the growth of iron oxide nanoparticles. The spectrum exhibits all the previously observed bands of the silica structure with the addition of one more peak located at $570 \mathrm{~cm}^{-1}$, which, according to the literature, is an indication of the vibration of $\mathrm{Si}-\mathrm{O}-\mathrm{Fe}-\mathrm{O}$ [34]. The aforementioned results in relation to the EDS analysis (Figure 3) clearly denote the presence of iron oxide on the surface of the silica microspheres.

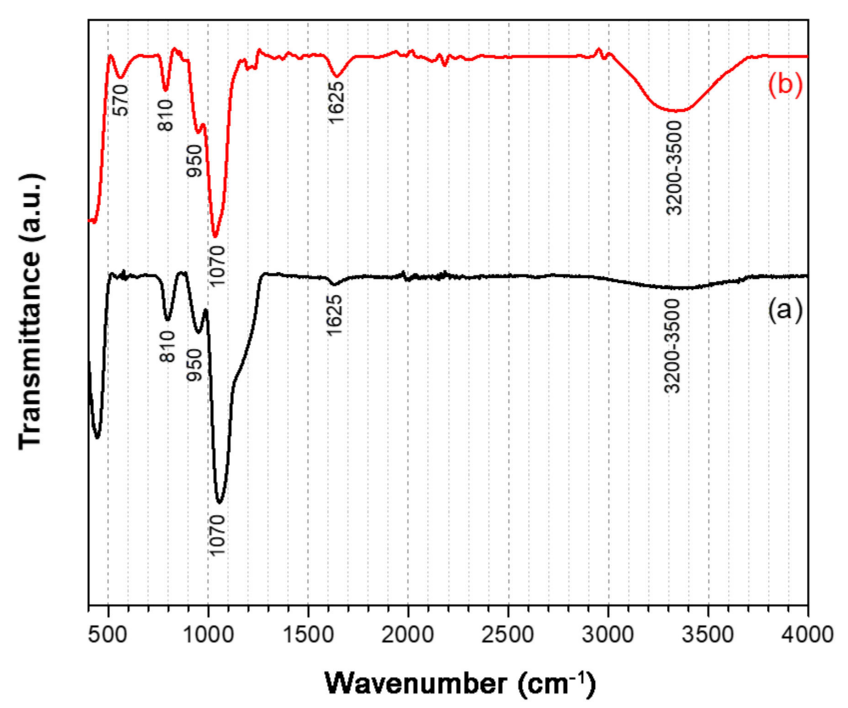

Figure 7. FT-IR spectra of (a) pristine silica microspheres and (b) $\mathrm{Fe}^{+}$-functionalized silica microspheres.

\subsection{Microwave Absorption Evaluation}

The triggerable properties of the produced microparticles were estimated via microwave absorption evaluation. Figure 8 illustrates the samples tested (top) and the results of the microwave absorption and the subsequent heat production of each measured sample, as identified by the IR thermo-camera (bottom). Four measurements took place: (a) an empty vial as a control, (b) silica microspheres, (c) pristine commercial MWCNTs and (d) $\mathrm{SiO}_{2} @ \mathrm{CNTs}$. Each sample was microwave irradiated for $2 \mathrm{~min}$, and the temperature was measured inside the oven directly after microwave irradiation came to a halt; subsequently, the oven was left to cool for $5 \mathrm{~min}$ before starting the next measurement. On the top left of each image, the measured temperature is highlighted, whereas on the right side of each image, the temperature scale bar is presented. The bright yellow-red area observed in the background, which is present in all pictures, is the area directly below the microwave source. 


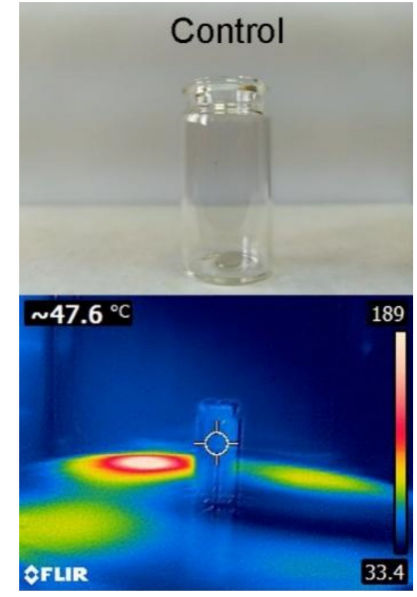

(a)

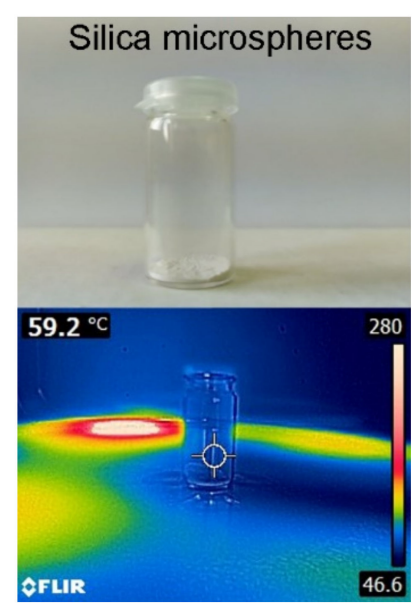

(b)

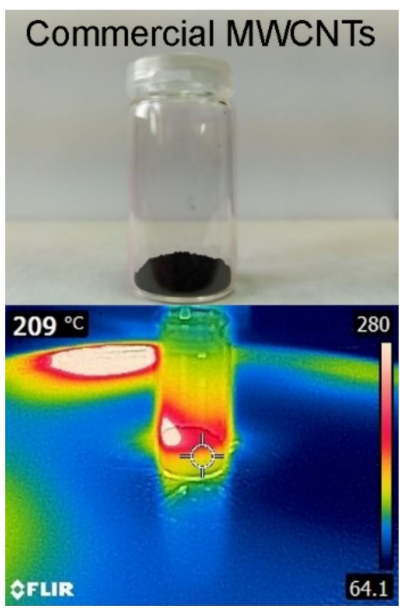

(c)

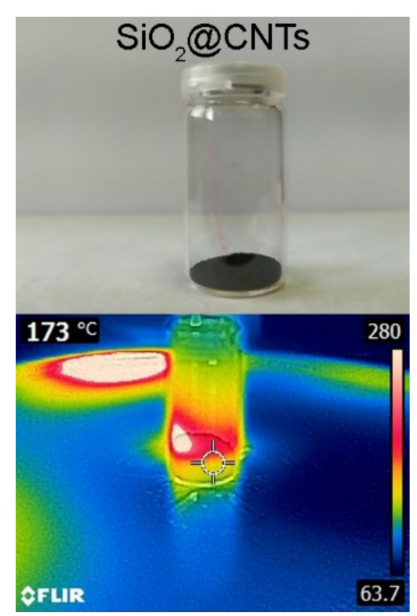

(d)

Figure 8. Photographs of the samples prior to (top) and after their microwave irradiation (bottom): (a) an empty vial as a control; (b) silica microspheres; (c) pristine commercial MWCNTs; (d) $\mathrm{SiO}_{2} @ C N T s$.

Taking into account the obtained results, it can be observed that the silica spheres exhibit a slightly increased temperature $\left(59.2^{\circ} \mathrm{C}\right)$ compared to the control sample, whereas the pristine commercial MWCNTs and the $\mathrm{SiO}_{2} @ \mathrm{CNT}$ exhibit elevated temperatures, $209^{\circ} \mathrm{C}$ and $173^{\circ} \mathrm{C}$, respectively. Furthermore, it can be seen that there is an increase in the temperature scale bar limits, from the control to the rest of the samples, from $33.4-189{ }^{\circ} \mathrm{C}$ up to $64-280^{\circ} \mathrm{C}$, indicating that the silica microspheres, pristine commercial MWCNTs and the hybrid $\mathrm{SiO}_{2} @ \mathrm{CNT}$ contribute to heat production. On the other hand, it may be remarked that despite the fact that the $\mathrm{SiO}_{2} @ \mathrm{CNT}$ contain the same amount of MWCNTs as the pristine commercial MWCNTs, there is a difference in the observed temperature $\left(\sim 30^{\circ} \mathrm{C}\right)$. This variance in temperature can be attributed to the difference between the $\mathrm{I}_{\mathrm{d}} / \mathrm{I}_{\mathrm{g}}$ ratios of the MWCNTs, as observed in the Raman analysis (Figure 5). It has been previously reported that morphological factors such as structural defects, aspect ratio, specific surface area and purity are prevailing factors that dictate the microwave absorption of carbon nanotubes [35]. In addition, considering that defect-free SWCNTs have been previously microwave irradiated in a similar manner and their heat production was significantly higher, the above hypothesis can be further supported [10].

\subsection{The Outcome Analysis}

It can be seen that three different materials were used. Taking the literature into account, in a previous study, Argawal et al. utilized the floating catalyst approach (xylene/ferrocene mixture) to grow MWCNTs on silica microspheres of various sizes [36]. Their results demonstrated that the size of the silica microspheres has an impact on MWCNTs' growth, whereas smaller values of the $I_{d} / I_{d}$ ratio were obtained for microspheres with sizes larger than $490 \mathrm{~nm}$. In addition, Zhou et al. functionalized silica nanoparticles with iron chloride, using methane as a carbon source, and produced SWCNTs; for silica spheres larger than $500 \mathrm{~nm}$, the SWCNTs spanned across different silica spheres, as also described in our work (Figure 1), whereas for larger silica spheres, SWCNTs engulfed the surface of their individual silica microspheres [37]. Nevertheless, Wei et al. modified silicon wafers with planar silica micro-discs and produced MWCNTs with ordered, preferential growth on the surface of the silica discs, utilizing the floating catalyst approach (xylene/ferrocene mixture) [38].

Evidently, the obtained results in the presented work agree with the above-mentioned research outcomes, further supporting the hypothesis that the growth of CNTs on silica microparticle substrates is affected by the size and the available surface area of the substrate. Silica microparticle substrates larger than $500 \mathrm{~nm}$ produce MWCNTs with fewer structural defects (judging by the $I_{d} / I_{g}$ ratio), whereas the appropriate selection of the carbon source 
and catalyst, with either a floating catalyst (xylene/ferrocene mixture) or a supported catalyst $\left(\mathrm{Fe}^{+}\right.$- functionalized silica microspheres with methane or acetylene as a carbon source), yields CNTs with ordered and random growth, respectively. In our case, the functionalization conditions, such as the weight percentage of the silica microspheres and the iron chloride concentration in the functionalization medium, were crucial for the functionalization of the entire population of silica microspheres and the homogenous growth of MWCNTs on each microparticle. Table 1 tabulates the physical and chemical properties of the three different materials that were used in our work.

Table 1. Tabulated values of physical and chemical properties of the used materials.

\begin{tabular}{cccc}
\hline & $\begin{array}{c}\text { Commercial } \\
\text { MWCNTs }\end{array}$ & SiO $_{2}$ & SiO $_{2} @ \mathbf{C N T s}$ \\
\hline Physical Properties & & 350 (sphere) & - \\
\hline Size $(\mathrm{nm})$ & $25-35$ (tube diameter) & -40 & - \\
Zeta potential $(\mathrm{mV})$ & - & stable & - \\
Thermal degradation $\left({ }^{\circ} \mathrm{C}\right)$ & $450-600[39]$ & 59.2 & 2.0 \\
$\mathrm{I}_{\mathrm{d}} / \mathrm{I}_{\mathrm{g}}$ & 0.6 & \multicolumn{3}{c}{173.0} \\
Heat production $\left({ }^{\circ} \mathrm{C}\right)$ & 209.0 & $\mathrm{Fe} ;-\mathrm{OH}$ & \\
\hline Chemical Properties & inert & $49.2-\mathrm{O} ; 48.0-\mathrm{Si} ;$ & $37.0-\mathrm{C} ; 31.5-\mathrm{O} ;$ \\
\hline Surface functionalization & $94.1-\mathrm{C} ; 5.9-\mathrm{O}$ & $2.8-\mathrm{Fe}$ & $30.3-\mathrm{Si} ; 1.2-\mathrm{Fe}$ \\
\hline Composition $($ wt. $\%)$ & &
\end{tabular}

Regarding the microwave absorption of similar materials, according to the literature, the study of Hekmatara et al. investigated the microwave absorption properties of $\mathrm{Fe}_{3} \mathrm{O}_{4} / \mathrm{MWCNT}$ materials coated with silica. The results revealed increased microwave absorption compared to the uncoated sample $\left(\mathrm{Fe}_{3} \mathrm{O}_{4} / \mathrm{MWCNT}\right)$ [40]. In the work of $\mathrm{Xi}$ ang et al., MWCNT-fused silica composites were fabricated, demonstrating enhanced microwave attenuation properties [41]. Barron and his team performed experiments on the modification of ceramic particle surfaces with carbon nanotubes and silica. The exposure of the produced hybrid materials to a microwave source resulted in heating the carbon nanotubes [42]. In another study, Yuan et al. evaluated the microwave attenuation performances of $\mathrm{SiO}_{2}-\mathrm{MWCNTs}$ matrix composites. It was proved that the obtained materials had increased microwave absorption [43].

Taking into account the aforementioned results regarding microwave absorption, it may be remarked that the comparison of our results with the literature could possibly lead to misinterpretation. The reason is that, in our study, the applied method for microwave absorption was used to compare the hybrid material $\left(\mathrm{SiO}_{2} @ \mathrm{CNTs}\right)$ with the reference (MWCNTs) in order to approximately estimate the application performance.

\section{Conclusions}

Silica microspheres functionalized with iron chloride were fabricated and used for the growth of MWCNTs on their surface. The obtained hybrid structures consisted of $80 \mathrm{wt} . \%$ silica and $20 \mathrm{wt} . \%$ MWCNTs, with an average diameter of $50 \mathrm{~nm}$ and an average degree of structural defects. The $\mathrm{SiO}_{2} @ \mathrm{CNTs}$ structures after 2 min microwave irradiation (magnetron $700 \mathrm{~W}$ operating at $2.45 \mathrm{GHz}$ ) produced enough heat to increase the material's temperature from ambient temperature up to $173{ }^{\circ} \mathrm{C}$, compared to the pristine commercial MWCNTs, which exhibited a measured temperature of $209^{\circ} \mathrm{C}$. By correlating these results with similar research in the literature related to the growth of CNTs on silica microspheres, it can be assumed that the size of the silica microspheres, the functionalization process with iron chloride and the selected carbon source are key variables for the physical characteristics of the resulting MWCNTs in terms of morphology and structural defects. Evidently, these hybrid structures can be tailored by modulating the aforementioned variables in order to produce structures with specific microwave absorption potential, whereas the silica part 
has the potential to be further functionalized in order to provide chemical affinity with polymer resins, contrary to the chemical inertness of pristine MWCNTs. The results from this study could greatly benefit polymer technology and the coating industry.

Author Contributions: Conceptualization, P.K. and I.A.K.; methodology, P.K. and I.A.K.; software, P.K. and I.A.K.; validation, P.K., I.A.K. and C.A.C.; formal analysis, P.K. and I.A.K.; investigation, P.K. and I.A.K.; resources, P.K. and I.A.K.; data curation, P.K.; writing-original draft preparation, P.K. and I.A.K.; writing-review and editing, I.A.K. and C.A.C.; visualization, P.K. and I.A.K.; supervision, C.A.C.; project administration, C.A.C.; funding acquisition, C.A.C. All authors have read and agreed to the published version of the manuscript.

Funding: This research was funded by the HORIZON 2020 Collaborative project "DECOAT" (Recycling of coated and painted textile and plastic materials, Grant agreement no.: 814505).

Institutional Review Board Statement: Not applicable.

Informed Consent Statement: Not applicable.

Data Availability Statement: Data sharing not applicable.

Conflicts of Interest: The authors declare no conflict of interest.

\section{References}

1. Rhein, S.; Sträter, K.F. Corporate self-commitments to mitigate the global plastic crisis: Recycling rather than reduction and reuse. J. Clean. Prod. 2021, 296, 126571. [CrossRef]

2. Wypych, G. 3-mechanisms of adhesion loss. In Handbook of Adhesion Promoters; Wypych, G., Ed.; ChemTec Publishing: Scarborough, ON, Canada, 2018; pp. 45-53. [CrossRef]

3. Bandl, C.; Kern, W.; Schlögl, S. Adhesives for "debonding-on-demand": Triggered release mechanisms and typical applications. Int. J. Adhes. Adhes. 2020, 99, 102585. [CrossRef]

4. Banea, M. Debonding on Demand of Adhesively Bonded Joints: A Critical Review. Rev. Adhes. Adhes. 2019, 7, 33-50. [CrossRef]

5. Ferahian, A.-C.; Hohl, D.K.; Weder, C.; Montero de Espinosa, L. Bonding and Debonding on Demand with Temperature and Light Responsive Supramolecular Polymers. Macromol. Mater. Eng. 2019, 304, 1900161. [CrossRef]

6. Guo, D.; Xie, G.; Luo, J. Mechanical properties of nanoparticles: Basics and applications. J. Phys. D Appl. Phys. 2014, 47, 013001. [CrossRef]

7. Kartsonakis, I.A.; Goulis, P.; Charitidis, C.A. Triggerable Super Absorbent Polymers for Coating Debonding Applications. Polymers 2021, 13, 1432. [CrossRef] [PubMed]

8. Goulis, P.; Kartsonakis, I.A.; Charitidis, C.A. Synthesis and Characterization of a Core-Shell Copolymer with Different Glass Transition Temperatures. Fibers 2020, 8, 71. [CrossRef]

9. Gupta, N.; Gupta, S.M.; Sharma, S.K. Carbon nanotubes: Synthesis, properties and engineering applications. Carbon Lett. 2019, 29, 419-447. [CrossRef]

10. Imholt, T.J.; Dyke, C.A.; Hasslacher, B.; Perez, J.M.; Price, D.W.; Roberts, J.A.; Scott, J.B.; Wadhawan, A.; Ye, Z.; Tour, J.M. Nanotubes in Microwave Fields: Light Emission, Intense Heat, Outgassing, and Reconstruction. Chem. Mater. 2003, 15, 3969-3970. [CrossRef]

11. Strozzi, M.; Pellicano, F. Linear vibrations of triple-walled carbon nanotubes. Math. Mech. Solids 2017, 23, 1456-1481. [CrossRef]

12. He, X.Q.; Eisenberger, M.; Liew, K.M. The effect of van der Waals interaction modeling on the vibration characteristics of multiwalled carbon nanotubes. J. Appl. Phys. 2006, 100, 124317. [CrossRef]

13. Pantano, A.; Parks, D.M.; Boyce, M.C. Mechanics of deformation of single- and multi-wall carbon nanotubes. J. Mech. Phys. Solids 2004, 52, 789-821. [CrossRef]

14. Vázquez, E.; Prato, M. Carbon Nanotubes and Microwaves: Interactions, Responses, and Applications. ACS Nano 2009, 3 , 3819-3824. [CrossRef]

15. Wadhawan, A.; Garrett, D.; Perez, J.M. Nanoparticle-assisted microwave absorption by single-wall carbon nanotubes. Appl. Phys. Lett. 2003, 83, 2683-2685. [CrossRef]

16. Tobias, G.; Mendoza, E.; Ballesteros, B. Functionalization of Carbon Nanotubes. In Encyclopedia of Nanotechnology; Bhushan, B., Ed.; Springer: Dordrecht, The Netherlands, 2012; pp. 911-919. [CrossRef]

17. Stöber, W.; Fink, A.; Bohn, E. Controlled growth of monodisperse silica spheres in the micron size range. J. Colloid Interface Sci. 1968, 26, 62-69. [CrossRef]

18. Wang, X.; Huang, J.; Feng, H.; Li, J.; Pu, Z.; Yin, X. Facile preparation of the dendritic $\mathrm{Fe}_{3} \mathrm{O}_{4}$ with a core-shell microstructure in $\mathrm{SiO}_{2}-\mathrm{B}_{2} \mathrm{O}_{3}-\mathrm{Al}_{2} \mathrm{O}_{3}-\mathrm{CaO}-\mathrm{Fe}_{2} \mathrm{O}_{3}$ glass-ceramic system for enhanced microwave absorbing performance. J. Alloys Compd. 2021, 877, 160147. [CrossRef]

19. Green, M.; Liu, Z.; Xiang, P.; Liu, Y.; Zhou, M.; Tan, X.; Huang, F.; Liu, L.; Chen, X. Doped, conductive $\mathrm{SiO}_{2}$ nanoparticles for large microwave absorption. Light Sci. Appl. 2018, 7, 87. [CrossRef] 
20. Kainourgios, P.; Tziveleka, L.-A.; Kartsonakis, I.A.; Ioannou, E.; Roussis, V.; Charitidis, C.A. Silver Nanoparticles Grown on CrossLinked Poly (Methacrylic Acid) Microspheres: Synthesis, Characterization, and Antifungal Activity Evaluation. Chemosensors 2021, 9, 152. [CrossRef]

21. Yu, Z.; Chen, D.; Tøtdal, B.; Holmen, A. Effect of catalyst preparation on the carbon nanotube growth rate. Catal. Today 2005, 100, 261-267. [CrossRef]

22. Yue, R.; Chen, Q.; Li, S.; Zhang, X.; Huang, Y.; Feng, P. One-step synthesis of 1,6-hexanediamine modified magnetic chitosan microspheres for fast and efficient removal of toxic hexavalent chromium. Sci. Rep. 2018, 8, 11024. [CrossRef]

23. Chiani, E.; Azizi, S.N.; Ghasemi, S. PdCu bimetallic nanoparticles decorated on ordered mesoporous silica (SBA-15)/MWCNTs as superior electrocatalyst for hydrogen evolution reaction. Int. J. Hydrog. Energy 2021, 46, 25468-25485. [CrossRef]

24. Mansfield, E.; Kar, A.; Hooker, S.A. Applications of TGA in quality control of SWCNTs. Anal. Bioanal. Chem. 2010, 396, 1071-1077. [CrossRef] [PubMed]

25. Javed, M.; Abbas, S.M.; Hussain, S.; Siddiq, M.; Han, D.; Niu, L. Amino-functionalized silica anchored to multiwall carbon nanotubes as hybrid electrode material for supercapacitors. Mater. Sci. Energy Technol. 2018, 1, 70-76. [CrossRef]

26. Yudianti, R.; Indrarti, L.; Onggo, H. Thermal Behavior of Purified Multi Walled Carbon Nanotube. J. Appl. Sci. 2010, 10, 1978-1982. [CrossRef]

27. Silva, M.A.; Felgueiras, H.P.; de Amorim, M.T.P. Carbon based membranes with modified properties: Thermal, morphological, mechanical and antimicrobial. Cellulose 2019, 27, 1497-1516. [CrossRef]

28. Maddalena, R.; Hall, C.; Hamilton, A. Effect of silica particle size on the formation of calcium silicate hydrate [C-S-H] using thermal analysis. Thermochim. Acta 2019, 672, 142-149. [CrossRef]

29. Li, Z.Q.; Lu, C.J.; Xia, Z.P.; Zhou, Y.; Luo, Z. X-ray diffraction patterns of graphite and turbostratic carbon. Carbon 2007, 45, 1686-1695. [CrossRef]

30. Das, R.; Hamid, S.; Ali, M.; Ramakrishna, S.; Yongzhi, W. Carbon Nanotubes Characterization by X-ray Powder Diffraction-A Review. Curr. Nanosci. 2014, 11, 23-35. [CrossRef]

31. Iijima, S.; Ichihashi, T. Single-shell carbon nanotubes of 1-nm diameter. Nature 1993, 363, 603-605. [CrossRef]

32. Nordgreen, T.; Liliedahl, T.; Sjöström, K. Elemental Iron as a Tar Breakdown Catalyst in Conjunction with Atmospheric Fluidized Bed Gasification of Biomass: A Thermodynamic Study. Energy Fuels 2006, 20, 890-895. [CrossRef]

33. Azarshin, S.; Moghadasi, J.; Aboosadi, Z.A. Surface functionalization of silica nanoparticles to improve the performance of water flooding in oil wet reservoirs. Energy Explor. Exploit. 2017, 35, 685-697. [CrossRef]

34. Ahangaran, F.; Hassanzadeh, A.; Nouri, S. Surface modification of $\mathrm{Fe}_{3} \mathrm{O}_{4} @ \mathrm{SiO}_{2}$ microsphere by silane coupling agent. Int. Nano Lett. 2013, 3, 1-5. [CrossRef]

35. Savi, P.; Giorcelli, M.; Quaranta, S. Multi-Walled Carbon Nanotubes Composites for Microwave Absorbing Applications. Appl. Sci. 2019, 9, 851. [CrossRef]

36. Agrawal, S.; Kumar, A.; Frederick, M.J.; Ramanath, G. Hybrid Microstructures from Aligned Carbon Nanotubes and Silica Particles. Small 2005, 1, 823-826. [CrossRef]

37. Zhou, W.; Zhang, Y.; Li, X.; Yuan, S.; Jin, Z.; Xu, J.; Li, Y. Preferential Growth of Single-Walled Carbon Nanotubes on Silica Spheres by Chemical Vapor Deposition. J. Phys. Chem. B 2005, 109, 6963-6967. [CrossRef]

38. Wei, B.Q.; Vajtai, R.; Jung, Y.; Ward, J.; Zhang, R.; Ramanath, G.; Ajayan, P.M. Organized assembly of carbon nanotubes. Nature 2002, 416, 495-496. [CrossRef]

39. Mahajan, A.; Kingon, A.; Kukovecz, Á.; Konya, Z.; Vilarinho, P.M. Studies on the thermal decomposition of multiwall carbon nanotubes under different atmospheres. Mater. Lett. 2013, 90, 165-168. [CrossRef]

40. Hekmatara, H.; Seifi, M.; Forooraghi, K.; Mirzaee, S. Synthesis and microwave absorption characterization of $\mathrm{SiO}_{2}$ coated $\mathrm{Fe}_{3} \mathrm{O}_{4}$-MWCNT composites. Phys. Chem. Chem. Phys. PCCP 2014, 16, 24069-24075. [CrossRef]

41. Xiang, C.; Pan, Y.; Liu, X.; Sun, X.; Shi, X.; Guo, J. Microwave attenuation of multiwalled carbon nanotube-fused silica composites. Appl. Phys. Lett. 2005, 87, 123103. [CrossRef]

42. Gomez, V.; Dunnill, C.W.; Barron, A.R. A microwave cured flux for the adhesion of ceramic particles using silica coated carbon nanotubes. Carbon 2015, 93, 774-781. [CrossRef]

43. Wen, B.; Cao, M.-S.; Hou, Z.-L.; Song, W.-L.; Zhang, L.; Lu, M.-M.; Jin, H.-B.; Fang, X.-Y.; Wang, W.-Z.; Yuan, J. Temperature dependent microwave attenuation behavior for carbon-nanotube/silica composites. Carbon 2013, 65, 124-139. [CrossRef] 